\title{
Right heart thrombus: the importance of early intervention
}

\author{
Helen Cosgrove, Patrick Hyland-McGuire
}

\begin{abstract}
A case report of mobile, right heart thrombus in the accident and emergency (A\&E) department is presented. Though frequently associated with major pulmonary embolism, recognition is usually at postmortem examination. Detection of the presence of mobile thrombus in the right heart by early echocardiogram and prompt treatment may be life saving. Surgical or medical treatment options are dependent on local facilities. Early specialist involvement with a contingency plan in A\&E departments are advised. (F Accid Emerg Med 1999;16:291-292)
\end{abstract}

Keywords: right heart thrombus; pulmonary embolus; thrombolysis or thrombectomy?

Right heart thrombus is frequently associated with major pulmonary thromboembolism, but is usually only recognised at postmortem examination. We present a case report where the diagnosis was made promptly and consequent early involvement of specialists resulted in the patient's survival.

\section{Accident and Emergency Department, Derriford Hospital, Derriford Road, Plymouth, Devon PL6 8DH H Cosgrove P Hyland-McGuire \\ Correspondence to: Dr Cosgrove, Specialist Registrar. \\ Accepted 10 September 1998}

\section{Case report}

A 27 year old, overweight woman was brought to our accident and emergency (A\&E) department by ambulance. One month previously she had had a sore throat treated by her family doctor with antibiotics. Over the fortnight before being brought to the $\mathrm{A} \& \mathrm{E}$ department, she had become increasingly breathless and this had failed to respond to inhaled salbutamol or steroid. On the day of her admission her dyspnoea had suddenly worsened and she had developed right sided pleuritic chest pain. In addition she had three episodes of haemoptysis

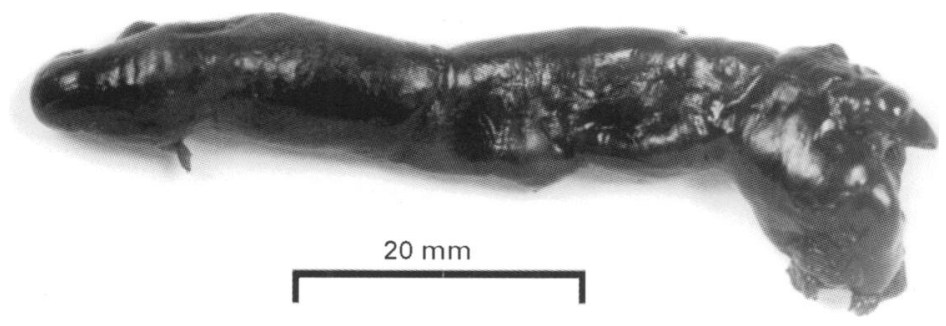

Figure 1 Serpiginous clot removed from right heart.

that day. Her only medication was the oral contraceptive pill. On arrival in the department she was obviously unwell. Her vital signs showed a pulse rate of 130 beats/min, a systolic blood pressure of $65 \mathrm{~mm} \mathrm{Hg}$, and a respiratory rate of 28 breaths/min. Her capillary refill time was greater than five seconds. Her chest was clear and with no pleuritic rub but a gallop rhythm was heard. Examination of her abdomen and pelvis was unremarkable.

Investigations revealed leucocytosis with a white cell count of $23.0 \times 10^{9} / 1$. Arterial blood gases and biochemistry were within normal limits. Electrocardiography showed a pattern of right heart strain and chest radiography showed enlarged, prominent pulmonary vessels and cardiomegaly. After initial resuscitation an emergency echocardiogram was performed in the $A \& E$ department which demonstrated a serpiginous mass prolapsing from the right atrium to the right ventricle through the tricuspid valve. There was associated torrential tricuspid regurgitation. The right side of the heart was fluid overloaded, but the left side appeared normal. There was a small pericardial effusion. An ultrasound examination of the abdomen and pelvis, though incomplete, did reveal absence of flow in both femoral veins. In addition it showed that the inferior vena cava was patent above the level of the renal pelvis.

A diagnosis of intracardiac thrombus prolapsing through the tricuspid valve was made and the patient was transferred directly to the cardiac surgery theatre for immediate surgery. At sternotomy, the patient arrested but was quickly put onto cardiopulmonary by-pass. A large clot was removed from the right atrium through a superior vena cava venotomy (fig 1). The right ventricle appeared to be clear of clot. Further exploration revealed further clot in the pulmonary artery (fig 2) that had the appearance of semiorganised thrombus and was occluding both the right and left pulmonary artery. This was successfully evacuated. The patient was successfully weaned off cardiopulmonary by-pass and sent to the intensive care unit. She was discharged home eight days later with appropriate anticoagulation.

Echocardiography before discharge showed good function in both ventricles, although the right was mildly dilated. The pulmonary artery was mildly dilated but appeared free of clot. There was low grade mitral and tricuspid regurgitation. A ventilation perfusion scan showed defects consistent with a high probability of multiple pulmonary emboli. Anticoagulation for a nine month period was started on the advice of the haematologist and full 


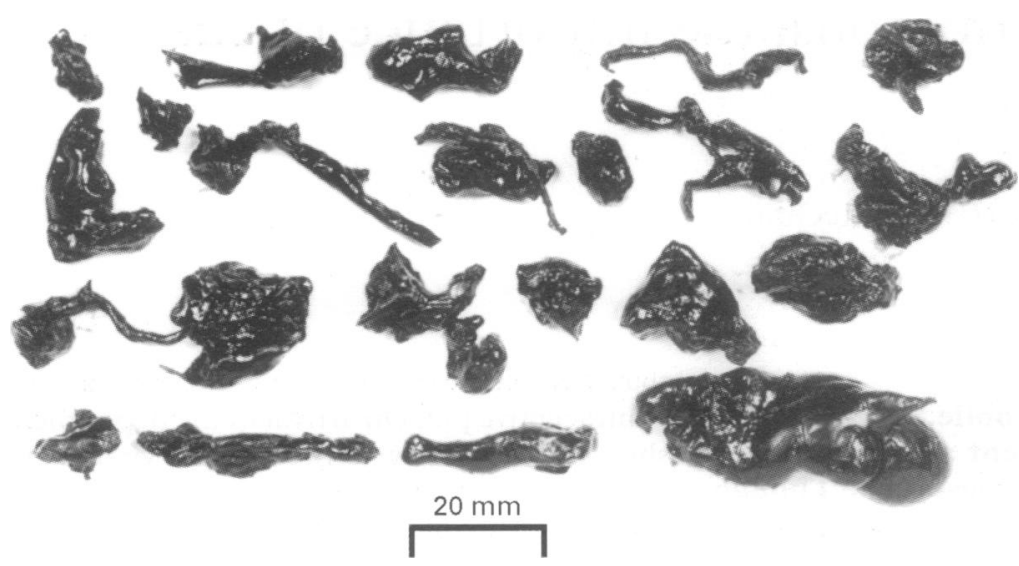

Figure 2 Clot removed from pulmonary arteries.

investigation for underlying coagulapathy was planned after this period.

\section{Discussion}

Mobile right heart thrombus is a well recognised association with acute thromboembolic disease. The incidence of this association is estimated to be in the order of $7 \%$ to $11 \%{ }^{1}$ Typically the heart thrombus is unstable and migration into the pulmonary artery is often fatal. ${ }^{2}$ Mortality rates of mobile right heart thrombus have been reported to be as high as $40 \%$. $^{1}$ For this reason some authors would advocate echocardiography in all cases of suspected pulmonary embolus where practicable. ${ }^{1}$ Even if the diagnosis is confirmed management of mobile right heart thrombus is controversial. ${ }^{13}$ Various authors favour anticoagulation, thrombolysis, or thrombectomy. ${ }^{3}$ Although all authors agree that mobile right heart thrombus needs emergency treatment, for practical reasons in the UK this will depend on local facilities. Emergency surgery with cardiopulmonary by-pass is not available in most district general hospitals unlike thrombolysis. In two reported series involving 14 patients ${ }^{1}$ and five patients ${ }^{3}$ respectively, the authors conclude that surgical removal of right heart thrombus is preferable to thrombolysis. A review of 49 cases revealed that $50 \%$ of patients treated medically died but only $15 \%$ of those treated surgically died. ${ }^{4}$ Nevertheless, there has since been anecdotal evidence that thrombolysis with recombinant tissue plasminogen activator is effective for right heart thrombosis. ${ }^{5}$ The proponents of early surgery quote the much lower mortality rates associated with operative removal of the thrombus, when compared with anticoagulation alone to support their case. ${ }^{1}$ Comparing thrombolysis with surgery is confounded by a lack of supporting data. Much of the literature is anecdotal. When thrombolysis is administered clinical improvement in patients is reported after approximately four hours. ${ }^{5}$ The advocates of surgery say that the inherent haemodynamic instability of these patients and their risk of sudden death makes delays of this order unacceptable. Our patient illustrated this point and indeed arrested at the start of the operation. We do not think that she would have survived had she not been in an area with rapid access to cardiopulmonary by-pass.

\section{Conclusions}

The possibility of mobile right heart thrombus should be considered in all cases of massive pulmonary embolism. If confirmed by echocardiography, prompt and aggressive treatment is warranted. Whether treatment is medical or surgical will depend largely on local facilities, but a contingency plan should be in place in all A\&E Departments.

We thank Mr J Kuo, Consultant Cardiothoracic Surgeon, for his permission to report on this patient.

Conflict of interest: none.

Funding: none.

1 Chapoutot L, Nazeyrollas P, Metz D, et al. Floating right heart thrombi and pulmonary embolism: diagnosis, outcome and therapeutic management. Cardiology 1996;87:169-74

2 Chakko S, Richards F. Right heart cardiac thrombi and pulmonary embolism. Am $\mathcal{F}$ Cardiol 1987;59:195-6.

3 Cameron J, Pohlner PG, Stafford EG, et al. Right heart thrombus: recognition, diagnosis and management. $\mathcal{f} \mathrm{Am}$ Coll Cardiol 1985;5:1239-43.

4 Farfal Z, Schecter M, Rath S, et al. Review of echocardiographically diagnosed right heart entrapment of pulmonary emboli-in-transit with emphasis on management. Am Heart 于1 1987;113:171-8.

5 d'Invernois C, Le Metayer P, Fisher B, et al. Life-threatening pulmonary embolism with right heart thrombus. Chest 1994;105:1291-2. 